Draft Version OCtober 29, 2018

Preprint typeset using $\mathrm{LAT}_{\mathrm{E}} \mathrm{X}$ style emulateapj v. 08/22/09

\title{
GRAVITATIONAL WAVE RECOIL AND THE RETENTION OF INTERMEDIATE MASS BLACK HOLES
}

\author{
Kelly Holley-Bockelmann ${ }^{1}$, Kayhan Gültekin ${ }^{2}$, Deirdre Shoemaker ${ }^{1}$, Nico Yunes ${ }^{1}$ \\ Draft version October 29, 2018
}

\begin{abstract}
During the inspiral and merger of a binary black hole, gravitational radiation is emitted anisotropically due to asymmetries in the merger configuration. This anisotropic radiation leads to a gravitational wave kick, or recoil velocity, as large as $\sim 4000 \mathrm{~km} \mathrm{~s}^{-1}$. We investigate the effect gravitational recoil has on the retention of intermediate mass black holes (IMBH) within Galactic globular clusters. Assuming that our current understanding of IMBH-formation is correct and yields an IMBH-seed in every globular cluster, we find a significant problem retaining low mass IMBHs $\left(\lesssim 1000 \mathrm{M}_{\odot}\right)$ in the typical merger-rich globular cluster environment. Given a uniform black hole spin distribution and orientation and a Kroupa IMF, we find that at most $3 \%$ of the globular clusters can retain an IMBH larger than $1000 M_{\odot}$ today. For a population of black holes that better approximates mass loss from winds and supernovae, we find that $16 \%$ of globulars can retain an IMBH larger than $1000 \mathrm{M}_{\odot}$. Our calculations show that if there are black holes of mass $M>60 \mathrm{M}_{\odot}$ in a cluster, repeated IMBH-BH encounters will eventually eject a $M=1000 \mathrm{M}_{\odot}$ IMBH with greater than $30 \%$ probability. As a consequence, a large population of rogue black holes may exist in our Milky Way halo. We discuss the dynamical implications of this subpopulation, and its possible connection to ultraluminous X-ray sources (ULXs).

Subject headings: black hole physics — galaxies: nuclei — gravitation — gravitational waves relativity
\end{abstract}

\section{INTRODUCTION}

Ample observational evidence exists for two types of black holes: stellar mass (BH), with $10 \mathrm{M}_{\odot} \lesssim m \lesssim 10^{2} \mathrm{M}_{\odot}$, and supermassive (SMBH), with $m \gtrsim 10^{6} \mathrm{M}_{\odot}$ (e.g., Kormendy \& Richstone (1995)). Although the existence of a third black hole class is still under debate, there are observational hints for intermediate mass black holes (IMBHs) as well, with masses $10^{2} \quad \mathrm{M}_{\odot} \lesssim m \lesssim 10^{5} \mathrm{M}_{\odot}$ (e.g., Gebhardt et al. (2005); Gerssen et al. (2002); Filippenko \& Ho (2003); Heggie et al. (2006b); Trenti et al. (2007c.a); Trenti (2006); Ulvestad et al. (2007); c.f. Baumgardt et al. (2003) for an alternative view.) These IMBHs may form within dense star clusters and may be the best explanation for ultraluminous X-ray sources (ULXs) in young star forming regions (Fabbiano 1989; Roberts \& Warwick 2000; Ptak \& Colbert 2004; Fabbiano \& White 2006) and in nearby extragalactic star clusters.

If the observational case for IMBHs is still unclear, the exact formation mechanism is perhaps less constrained. Within a globular cluster, there are currently three plausible IMBH formation theories: stellar runaway; compact object mergers; and growth from Population (Pop) III remnants (cf. van der Marel (2004) for a review.) Each of these mechanisms starts with very different initial conditions, and the first two require the high stellar densities of a globular or stellar cluster environment. Thus, growing IMBHs over the ensemble of observed globular

\footnotetext{
${ }^{1}$ IGPG, Center for Gravitational Wave Physics, The Pennsylvania State University, University Park, PA 16802, USA Email: kellyhb@gravity.psu.edu, deirdre@gravity.psu.edu, yunes@gravity.psu.edu

${ }^{2}$ Department of Astronomy, University of Michigan, Ann Arbor, MI, 48109, USA Email:kayhan@umich.edu
}

clusters may require more than one single mechanism. We briefly introduce these mechanisms below.

Recent simulations of core collapse in stellar clusters have shown that stellar collisions could induce rapid stellar runaway growth of an $\mathcal{O}\left(10^{3} \mathrm{M}_{\odot}\right)$ IMBH in as little as 3 Myr (Portegies Zwart \& McMillan 2002; Gürkan et al. 2006; (Freitag et al. 2006); see also (Portegies Zwart et al. 2004). However, this runaway growth process requires number densities higher than $\mathcal{O}\left(10^{6} / \mathrm{pc}^{3}\right)$ and few (if any) globular clusters in the Milky Way are this dense today. Of course, globular clusters are thought to be more dense at formation (Trenti et al. 2007b; Heggie et al. 2006a), but calculations still suggest that the runaway process may only occur in about $20 \%$ of the Milky Way globular cluster system (Baumgardt et al. 2005). By using less extreme central densities and including the effect of primordial binaries, simulations have shown that instead of a single IMBH, stellar runaways generate two or more black holes with masses $\sim 500 M_{\odot}$ (Gürkan et al. 2006). When these large black holes merge they form a single IMBH and a strong gravitational wave signal for the Laser Interferometer Space Antenna, or LISA, a planned gravitational wave observatory set to launch in the next decade(Fregeau et al. 2006; Gürkan et al. 2006).

While runaway star collisions seem to be the preferred IMBH formation channel within dense systems, in systems where the relaxation time is long, stellar mass $B H$ collisions may be a more likely growth mechanism. Here the initial seed IMBH forms naturally from a heavier than average black hole, perhaps a supernova remnant with $\sim 250 \mathrm{M}_{\odot}$ (Wise \& Abel 2005$)$. Then, stellar mass $\mathrm{BHs}$ are captured by the initial seed, and the resulting binary hardens through interactions with other stellarmass objects. Although these interactions risk ejecting 
the $\mathrm{BH}$ supply before significant growth occurs, one way to funnel more BHs to the growing IMBH is through 4body collisions (O'Leary et al. 2006; Miller \& Hamilton 2002; Gültekin et al. 2004).

Significantly higher mass Pop III stars may yield IMBHs directly. If a Pop III protostar has a mass of $10^{5} M_{\odot}$, it will be gravitationally unstable and will collapse to an IMBH before it even enters the main sequence (Baumgarte \& Shapiro 1999; Shibata \& Shapiro 2002). The probability of an IMBH forming from such a massive Pop III star certainly depends on the initial stellar mass function (IMF), as well as highly uncertain details of zero-metallicity stellar evolution. However, there has been some suggestions that the IMF in the early universe is quite top-heavy (Schneider et al. 2002; Abel et al. 2002), and that stellar mass loss is negligible (Frver et al. 2001; Heger et al. 2003). These suggestions imply that BHs in a proto globular cluster environment are more massive than those formed in more recent times (van der Marel 2004). On the other hand, Pop III star formation is thought to take place in dark matter overdensities at $z \sim 12-20$ (Madau \& Rees 2001) and only the most massive globular clusters are thought to be embedded in a significant enough dark matter overdensity to allow for Pop III formation.

However the IMBH forms, for the first $\sim 0.5$ Gyr after formation it is dynamically active (Spitzer 1987). Due to mass segregation, the initial environment around an $\mathrm{IMBH}$ in a globular cluster is especially rich in BHs (Fregeau et al. 2002). While many of these BHs are quickly ejected by few body interactions with the IMBH, enough remain to subject the IMBH to $10 \mathrm{~s}-100 \mathrm{~s}$ mergers with BHs in the primordial globular cluster system (Portegies Zwart \& McMillan 2000; O'Leary et al. 2006; Gültekin et al. 2004).

In light of general relativistic black hole merger simulations, surviving this IMBH-BH merger epoch may be difficult. Recent advances in numerical relativity have at last pinned down the dynamics of black hole mergers - simulating the coalescence, merger, and ringdown of equal-mass circular non-spinning binary black holes in full general relativity (Pretorius 2005; Campanelli et al. 2005; Baker et al. 2006). In the past year, more astrophysically relevant numerical simulations, including spins and unequal masses, have been published by several groups.

One of the most exciting results of general relativity for structure formation is that binary black hole systems strongly radiate linear momentum in the form of gravitational waves during the plunge phase of the inspiral. This radiation directly results from an asymmetry in the orbital configuration and can generically yield a gravitational wave "kick" velocity as fast as $4000 \mathrm{~km} \mathrm{~s}^{-1}$ (Gonzalez et al. 2007b; Campanelli et al. 2007b). Even typical kick velocities $\left(\sim 200 \mathrm{~km} \mathrm{~s}^{-1}\right)$ are interestingly large when compared to the escape velocity of an average globular cluster $\left(\sim 50 \mathrm{~km} \mathrm{~s}^{-1}\right)$ (Webbink 1985; Favata et al. 2004; Merritt et al. 2004). Hence, regardless of how an IMBH forms, the biggest challenge may be to determine how a globular cluster retains them in the face of a repeated onslaught of gravitational wave kicks from mergers with other black holes. Of course if IMBHs are formed through mergers of stellar-mass black holes, the formation mechanism itself needs to be able to account for these large kicks as well.

In this paper we explore IMBH retention within young globular clusters after collisions with BHs. We calculate the retention probability under a variety of assumptions for the initial IMBH seed mass, the BH mass distribution, and the initial spin distributions. In addition, for the mass range encompassed by the IMBH formation channels listed above (stellar runaway, stellar-mass $\mathrm{BH}$ collisions, Pop III stars), we simulate the IMBH survival probability after merging with the available BHs over a collision timescale. Given the known current structure of the Milky Way globular cluster system, we can then estimate the fraction of globular clusters that may have allowed a particular IMBH formation channel. Combining these two key estimates, we can determine the maximum expected number of Milky Way globular clusters that could have retained their IMBHs. We outline the approach in Sec. 2, present the results in Sec. 3 and discuss the caveats, implications, and future directions in Sec. 4.

\section{ASSIGNING KICKS}

Gravitational recoil estimates of binary black hole mergers have been addressed using both semi-analytic methods in the unequal-mass, non-spinning case (Fitchett 1983; Favata et al. 2004; Damour \& Gopakumar 2006; Blanchet et al. 2005; Sopuerta et al. 2006) and numerical methods in more general, spinning, unequal mass scenarios (Herrmann et al. 2007; Baker et al. 2006; Gonzalez et al. 2006; Herrmann et al. 2007; Koppitz et al. 2007; Gonzalez et al. 2007a; Campanelli et al. 2007a,c; Tichy \& Marronetti 2007). For non-spinning binaries, the most comprehensive numerical study of kicks is that of Gonzalez et al. (2006), which was found in agreement with the semi-analytic estimates of (Sopuerta et al. 2006), where a maximum kick velocity of $\sim 175 \pm 10 \mathrm{~km} \mathrm{~s}^{-1}$ was obtained for mass ratio $\left.q=M_{1} / M_{2} \sim 0.36 \pm 0.02\right)$.

The gravitational recoil is expected to increase with increasing spin (Redmount \& Rees 1989; Whitbeck 2006), and this behavior has been confirmed by several numerical relativity groups. Numerical simulations have demonstrated that the radiative linear momentum loss predicted by Post-Newtonian studies (Thorne 1980; Kidder 1995) can be used to fit numerical results, yielding a generalized formula for the recoil velocity as a function of the individual black hole's spin, initial orientation, phase at merger and mass ratio. We here adopt the parameterized fit of Campanelli et al. (2007a) adding the expected $(1+e)$ contribution for eccentric orbits (Sopuerta et al. 2007) to yield the following formula:

$$
v_{\text {kick }}=(1+e)\left[\hat{x}\left(v_{m}+v_{\perp} \cos \xi\right)+\hat{y} v_{\perp} \sin \xi+\hat{z} v_{\|}\right]
$$

where

$$
\begin{gathered}
v_{m}=A \frac{q^{2}(1-q)}{(1+q)^{5}}\left[1+B \frac{q}{(1+q)^{2}}\right], \\
v_{\perp}=H \frac{q^{2}}{(1+q)^{5}}\left(\alpha_{2}^{\|}-q \alpha_{1}^{\|}\right),
\end{gathered}
$$


and

$$
v_{\|}=K \cos \left(\Theta-\Theta_{0}\right) \frac{q^{2}}{(1+q)^{5}}\left(\alpha_{2}^{\perp}-q \alpha_{1}^{\perp}\right),
$$

where the fitting constants are $A=1.2 \times 10^{4} \mathrm{~km} \mathrm{~s}^{-1}$, $B=-0.93, H=(7.3 \pm 0.3) \times 10^{3} \mathrm{~km} \mathrm{~s}^{-1}$, and $K=$ $(6.0 \pm 0.1) \times 10^{4} \mathrm{~km} \mathrm{~s}^{-1}$, while the subscripts 1 and 2 refer to the first and second $\mathrm{BH}$ respectively. The unit vectors $(\hat{x}, \hat{y})$ are orthogonal to each other and span the initial orbital plane, while $\perp$ and $\|$ stands for perpendicular and parallel to the orbital angular momentum. There are 4 fitting parameters: the mass ratio $q \equiv M_{2} / M_{1}$; the reduced spin parameter $\alpha_{i} \equiv S_{i} / M_{i}^{2}$, where $S_{i}$ is the spin angular momentum of $\mathrm{BH} i$; and the eccentricity $e$. In addition, there are 3 angles to specify the orientation of the merger: $\Theta$, the angle between the "in-plane" component of $\delta^{i} \equiv\left(M_{1}+M_{2}\right)\left(S_{2}^{i} / M_{2}-S_{1}^{i} / M_{1}\right)$ and the infall direction at merger; $\Theta_{0}$, the angle between $\delta^{i}$ and the initial direction of motion; and $\xi$, the angle between the unequal mass and spin contribution to the recoil in the orbital plane. The merger phase within the orbital plane may also play a role, but it is not included explicitly in this fit. The recoil velocities as given in Eq. (4) are plotted in Fig. 1 as a function of mass ratio and spin parameter.

In order to compute the probability that an IMBH remains in its globular cluster, our calculation shall proceed as follows. First we begin by assuming that an IMBH has formed within a globular cluster with a particular initial mass, $M_{\mathrm{IMBH}}$, which we shall vary. Second, we further assume a certain mass distribution for the BHs in the vicinity of the IMBH, which we shall also vary. Third, we subject this IMBH to a number of mergers expected within a proto globular cluster environment that we shall describe below. Finally, we determine the probability that the kick velocity for the IMBH has remained below the canonical globular cluster escape velocity $\left(50 \mathrm{~km} \mathrm{~s}^{-1}\right)$ during the entire chain of mergers.

Even in the absence of an IMBH, BHs eject themselves from globular clusters via standard few-body interactions on a timescale of $\sim 1$ Gyr after the onset of mass segregation (Kulkarni et al. 1993; Sigurdsson \& Hernquist 1993; Portegies Zwart \& McMillan 2000; O'Leary et al. 2006). Therefore, due to such Newtonian few-body interactions, the supply of BHs is eventually depleted. With an IMBH, however, this process speeds up impressively, as ejections by interactions with an IMBH become the dominant source of stellar-mass BH ejections (Gültekin et al. 2006). As in most few-body interactions, the ejection of one object tightens the orbit of a remaining bound pair, in this case an IMBH-BH binary - and after several subsequent ejections, the IMBH-BH binary merges. Soon after all the BHs have been evacuated, the short epoch of IMBH-BH mergers ends.

Within this theoretical framework, it is possible to construct a fiducial number of mergers for a proto globular cluster. This number can be written as

$$
N_{\text {merge }}=\frac{N_{\text {BHs }}}{n_{\text {ejections } / \text { merger }}} .
$$

Gultekin et al. 2006 predict $N_{\text {merge }} \sim 25$ per IMBH, and we adopt this for the fiducial number of mergers that the IMBH encounters. Although we do vary this parameter in figure 2, the dependence of the retention probability on the number of mergers is relatively minor, since the IMBH grows in mass over each merger and the kick velocity increases with increasing mass ratio.

In order to assign a kick velocity to each of the mergers, we choose the orientation, spin, mass, and eccentricity. We outline the assumptions made for each distribution below. Let us first discuss the issue of the initial spin orientation. Hydrodynamic interactions between a gas disk and a black hole binary are believed to align the spin directions to the angular momentum axis of the binary orbital plane in many active galaxies (Bogdanovic et al. 2007). However, the environment of a globular cluster is not particularly gas-rich, so there is no ab initio reason to expect the black hole spins to be aligned. We therefore assume an isotropic distribution of orientation angles for each encounter.

Let us now discuss the choice of spin magnitude. Most theories predict a non-zero spin for a black hole produced via stellar runaway (Rees \& Volonteri 2007) or from a supernovae remnant (Fryer et al. 2001). If an IMBH started with zero spin, a merger is likely to spin up the remnant through transfer of orbital to spin angular momentum (Gammie et al. 2004). However, a Kerr black hole can spindown when magnetic field lines thread through the ergosphere to magnetically brake the system (Blandford \& Znajek 1977), provided there is a gaseous disk around the remnant. Taking all these consideration into account, we shall explore three cases: (1) the spin magnitude is selected from a uniform initial spin distribution (fiducial case); (2) the initial spin magnitude of the IMBH seed is set to $0.998 M_{\mathrm{IMBH}}^{2}$; and (3) the spin is initially set to zero. We assume the spin of the secondary $\mathrm{BH}$ to be randomly selected from a distribution of $[0,0.998] M_{\mathrm{sec}}^{2}$, where $M_{\mathrm{sec}}$ is the mass of the secondary BH. Since these stellar-mass BHs originate as a supernova remnant, though, the spin is likely to be high (Heger et al. 2003). Therefore, since the kick velocity increases with increasing spin, this shall lead to a conservative survival probability for each merger tree.

The survival probability shall be studied as a function of the initial mass of the seed IMBH. We choose a range of $10-3000 M_{\odot}$ to encompass the plausible IMBH formation channels and relevant masses involved. For the three formation channels discussed in $\S 1$, the seed masses are likely to be the following: $\sim 1000 M_{\odot}$ for a single stellar runaway (Portegies Zwart et al. 2004); $\sim 100 M_{\odot}$ for the stellar mass BH collision channel, where the seed IMBH is produced by a massive supernova remnant or a small stellar runaway (Heger et al. 2003); and $\sim 200-400 M_{\odot}$ for Pop III remnants within a dense globular cluster (Wise \& Abel 2005; Madau \& Rees 2001).

One of the biggest uncertainties is determining a proper distribution for the secondary BH masses. As figure 2 shows, the retention probability depends strongly on the mass ratio between the IMBH and BH. Theoretical black hole mass distributions from solar metallicity field populations tend to peak strongly around $10 M_{\odot}$ (Fryer \& Kalogera 2001). There are, however, several strong competing effects in a primordial globular cluster that can change this distribution (e.g., low metallicity stellar evolution, high binary fraction, stellar colli- 

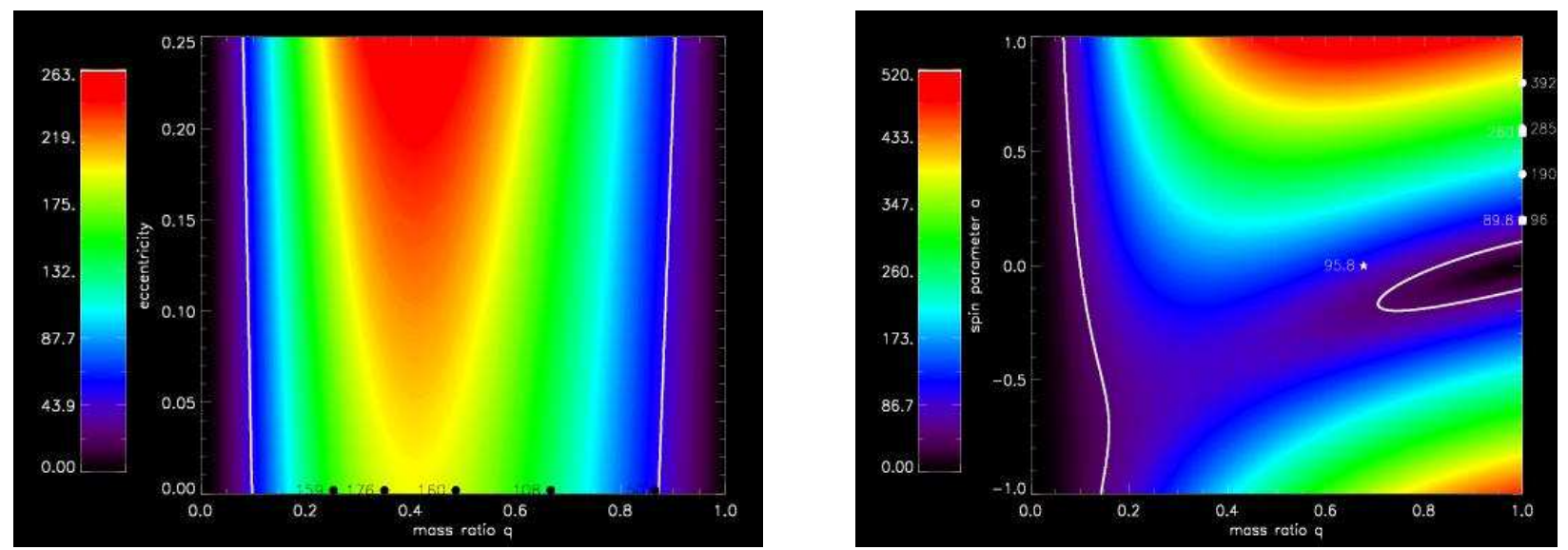

FIG. 1.- Left: The gravitational wave kick velocity from a non-spinning black hole merger as a function of mass ratio and eccentricity. The white contour marks $50 \mathrm{~km} \mathrm{~s}^{-1}$, the escape velocity of a typical globular cluster. Overlaid are data from numerical relativity merger simulations (Gonzalez et al. 2006) (black dots on zero-eccentricity line.) Note that most of this plane has not been confirmed by direct numerical experiment, but has been extrapolated from semi-analytic studies (Sopuerta et al. 2007). In this plane, only mergers with the largest mass ratios will remain in the globular cluster, which argues for a very high-mass initial seed. Right: The gravitational wave kick velocity from spinning black hole mergers on a circular orbit as a function of spin magnitude and mass ratio. Here, the spins of the black hole are anti-aligned to one another and are perpendicular to the orbital plane of the binary black hole before merger. Note that other spin orientations yield even higher kicks. As in the previous figure, the $50 \mathrm{~km} \mathrm{~s}^{-1}$ contour is overplotted, as well as selected results from full numerical relativity simulations: squares are from Koppitz et al. (2007); circles are from Herrmann et al. (2007), and star is from Brügmann et al. (2004). This figure suggests that globular clusters can retain IMBHs only when they merge with other BHs with low spin and high mass ratio.

sions, mass segregation, and natal kicks). For our fiducial experiment, we assume that the secondary masses are selected from a Kroupa IMF with an upper mass cutoff of $120 M_{\odot}$ Kroupa 2001). Recall that this yields an average stellar mass of about $1 M_{\odot}$, much smaller than the suspected average stellar mass from a zero-metallicity environment. We further assume that each star above $10 M_{\odot}$ evolves directly to a $\mathrm{BH}$ with no mass loss. This simplified treatment gives us an average $\mathrm{BH}$ mass of $\sim 20 M_{\odot}$. Clearly, mass loss would decrease the average $\mathrm{BH}$ mass even in these low metallicity primordial globulars. Therefore, we used a more sophisticated black hole mass function that approximates the results of model C1 of Belczynski et al. (2006). This model includes the effect of mass loss from supernovae and winds for a population of stars with metallicity $Z=0.0001$, though it has fewer massive remnants from binary mergers (cf Figure 8 of Belczynski et al. (2006) ) than is expected for a primordial globular cluster.

Figure 3 demonstrates the effect on the kick velocity distribution between these two black hole mass functions for a $1000 M_{\odot} \mathrm{IMBH}$. Since the distribution of secondary masses is so uncertain, we demonstrated the effect of varying the mass ratio in Figure 2, For a near-solar metallicity stellar cluster, the entire population of black holes may be less than $M \lesssim 20 M_{\odot}$ (Fryer \& Kalogera 2001).

Finally, we must select the eccentricity distribution. If these IMBH-BH mergers were 2-body processes, we might expect the eccentricity of the orbit to be very nearly circular right before the black holes merge as gravitational radiation grinds away the orbital angular momentum (Peters \& Mathews 1963). However, few body encounters are much more common within a globular cluster because the interaction cross-section is much larger (Heggie \& Hut 2003). Therefore, many BHs are shepherded into mergers with an IMBH through ex- change with lower mass black holes (Miller \& Hamilton 2002), and the resulting eccentricity can be quite large (Gültekin et al. 2006). In fact, simulations have shown that rare interactions can yield mergers with $e>0.999$, and such a highly eccentric orbit can become even more eccentric through gravitational radiation emission (Peters \& Mathews 1963; Kennefick 1998). Therefore, though rare, highly eccentric binary black hole mergers can take place in astrophysically relevant systems. To assign eccentricities to each merger, we use the simulation results of Gültekin et al. (2006), which empirically characterizes the eccentricity distribution as a function of the mass ratio of the encounter. Note, though, that equation (11) is really only valid in the small eccentricity regime and, thus, it may be true that the kicks can be even higher than those studied here for such nearly radial orbits.

\section{IMBH SURVIVAL AND OCCUPATION FRACTION}

In order to determine the probability that an IMBH survives the short merger epoch after formation, our simulations consist of $10^{6}$ Monte Carlo realizations of an Nstep merger chain. The merger chains are tailored to mimic the initial conditions and encounters predicted by current IMBH formation theories within proto-globular and stellar cluster environments as described in the previous section.

During a merger, gravitational waves radiate not only linear momentum, but also angular momentum and energy or mass. Fully relativistic numerical simulations suggest that $\sim 25 \%$ of the angular momentum (defined at the innermost stable circular orbit) can be radiated during the merger (Pretorius 2005; Campanelli et al. 2005; Baker et al. 2006; Herrmann et al. 2007). In addition, the mass of the merger product is only $\sim 95 \%$ the mass of the two progenitor black holes (Pretorius 2005; Campanelli et al. 2005; Baker et al. 2006; Herrmann et al. 2007). Hence, after each step 

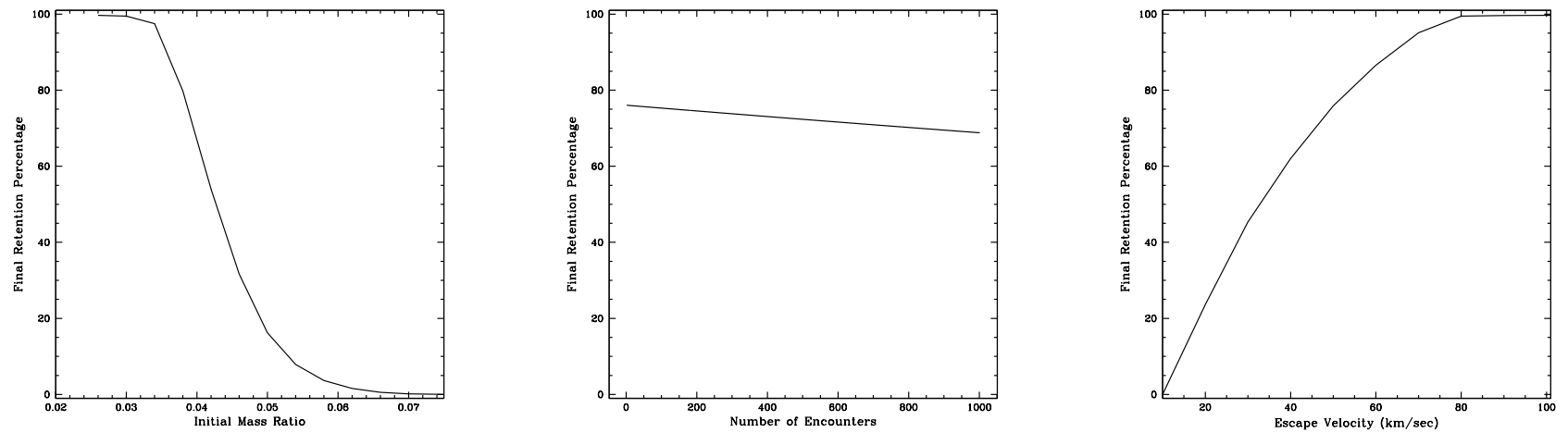

FIG. 2. - Left: Retention probability as a function of mass ratio of the interacting black holes. We assume an initial IMBH mass of $M_{\mathrm{IMBH}}=500 M_{\odot}$, an escape velocity of $50 \mathrm{~km} \mathrm{~s}^{-1}$, and a merger chain of 25 interactions. Mergers are chosen with random orientations and spin magnitudes. Middle: Retention probability as a function of the number of BH mergers. We assume $M_{\mathrm{sec}}=20 M_{\odot}, M_{\mathrm{IMBH}}=500 M_{\odot}$, and random orientations and spin magnitudes. Right: Retention probability as a function of the escape velocity of the host structure. We assume $M_{\mathrm{sec}}=20 M_{\odot}, M_{\mathrm{IMBH}}=500 M_{\odot}$, and random orientations and spin magnitudes.

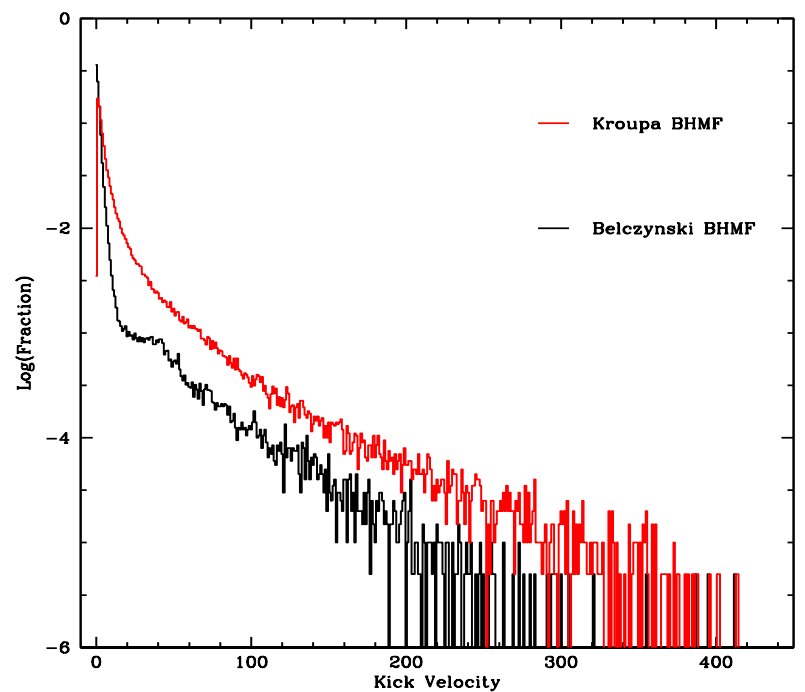

FIG. 3.- Fraction of encounters as a function of kick velocity for two black hole mass functions. We assume the IMBH has a mass of $1000 M_{\odot}$ and that the spin magnitudes and orientations are distributed uniformly.

within a particular merger tree, we adjust the total spin and mass of the merger remnant to account for these losses.

Figure 5 demonstrates the retention percentage after 25 mergers with black holes selected from a Kroupa IMF as a function of the initial IMBH mass. This figure indicates that retaining an IMBH of less than $1000 M_{\odot}$ occurs less than $33 \%$ of the time for the given distribution of black hole masses. While the large number of the lower-mass black holes dominate the total number of mergers, the rare mergers with massive stellar-mass black holes dominate the ejections - and this trend only strengthens as the IMBH mass increases. For example, figure 4 shows that for a $1000 \mathrm{M}_{\odot}$ seed, nearly all ejections come from black holes with mass $M>30 \mathrm{M}_{\odot}$ and that most come from those with mass $M \sim 70 \mathrm{M}_{\odot}$. Naturally, this implies that if $>30 M_{\odot}$ black holes are extremely rare in primordial globular clusters, the retention fraction increases dramatically (Frver \& Kalogera 2001),

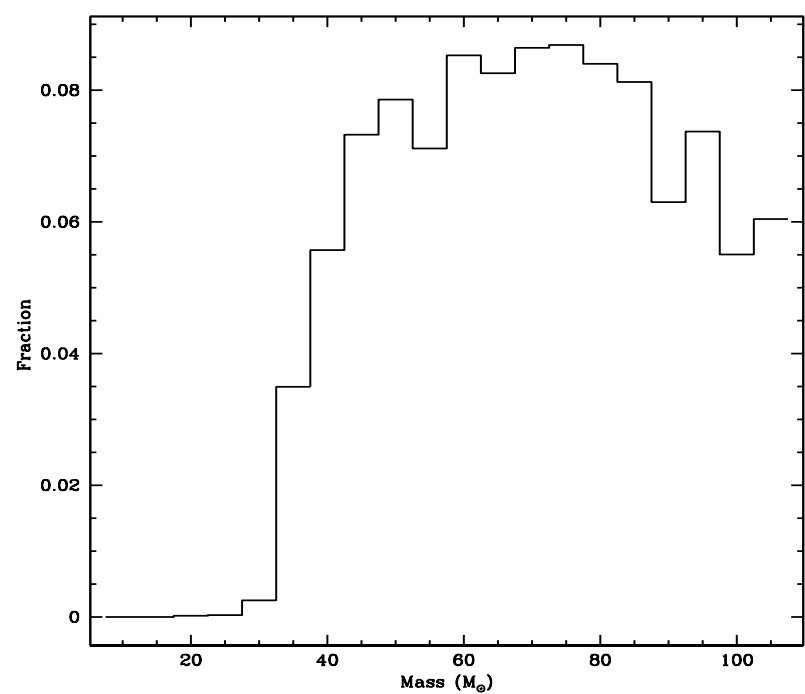

FIG. 4.- A histogram of the black hole masses that eject a $1000 M_{\odot}$ IMBH. In this experiment, we assumed a Kroupa IMF, and a uniform spin orientations and magnitude. Ejecting a $1000 M_{\odot}$ black hole is possible with masses as low as $20 M_{\odot}$, but the overwhelming majority of ejections result from black holes with masses $>50 M_{\odot}$.

making gravitational recoil ineffective in ejecting massive IMBHs. Figure 6 shows that it is easier to retain an IMBH of less than $1000 M_{\odot}$ with the shallower Belczynski black hole mass function, with only $30 \%$ ejected.

Recall that each formation channel is expected to take place in a particular proto-globular cluster environment (e.g., single runaways ought to form in very dense systems.) Since predictions of pre-core collapse clusters indicate that the densities are $\sim 10$ times higher than today, we can use current observations of the central luminosity density (Harris 1996) to infer the initial conditions of the globular cluster. Then, we can estimate on a caseby-case basis what IMBH formation channel might have been appropriate for each globular cluster. If we assume that every globular cluster forms an IMBH with a mass that is consistent with its presumed formation channel (see previous section), we can estimate the number of surviving IMBHs within the Milky Way globular cluster 


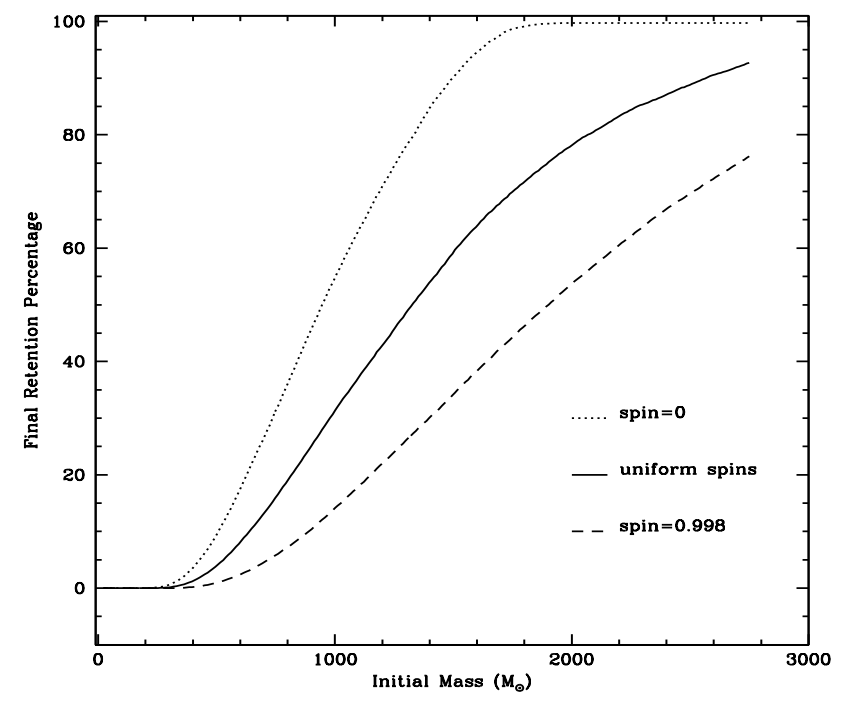

FIG. 5.- Percentage of black hole retention as a function of initial black hole mass within the Milky Way globular cluster system. A black hole is defined as 'retained' if it survives 25 collisions with a BH selected from a Kroupa IMF with random spin orientations. The three lines on this figure represent different assumptions for the spins of the black hole.

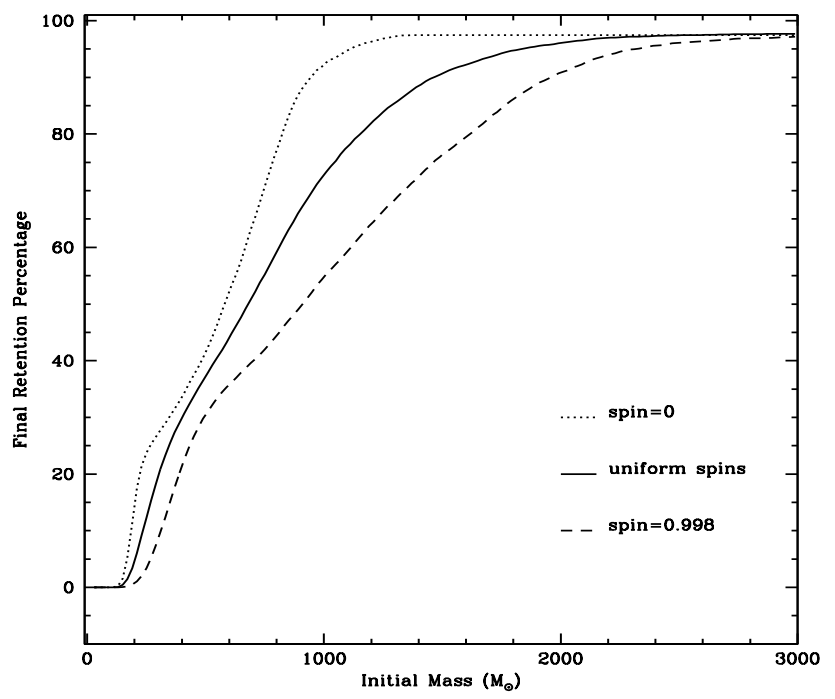

FIG. 6. - Percentage of black hole retention as a function of initial black hole mass within the Milky Way globular cluster system. In this experiment, we assumed a black hole mass function described by model C1 of Belczynski et al. (2006), and a uniform spin orientations and magnitude.

system. We find that $\sim 24 \%$ of the Milky Way globular clusters can host single runaways, while $\sim 25 \%$ may host multiple runaways. If, thereafter, the IMBH must survive 25 mergers selected from a Kroupa IMF, our study indicates that no more than 5 globular clusters have retained an IMBH, while our Belczynski black hole mass function yields $<25$ IMBH-embedded globulars.

Such conclusions can be split into the number that survive per formation channel. Doing so, we learn that the only way to retain IMBHs during the short $\mathrm{BH}$ merger phase is with one rather massive initial seed, such as those produced in a stellar runaway; any process that relies of the formation of $\lesssim 500 M_{\odot}$ black holes will be ineffective, as these are ejected from a globular cluster before forming an IMBH. One way to lower the retention mass threshold would be to increase the globular cluster escape velocity. We explore this effect in figure 2, where we observe that the escape velocity must reach $\sim 80 \mathrm{~km} \mathrm{~s}^{-1}$ to attain complete retention of $500 M_{\odot}$ black holes.

A BH mass function that is tipped more strongly to lower masses would also increase the retention rate - note that a Kroupa IMF with no mass loss yields an average $\mathrm{BH}$ mass of $\sim 20 M_{\odot}$, while allowing for mass loss will make high mass ratio mergers less likely. If there are no black holes $>20 M_{\odot}$ in clusters, then any IMBH seed larger than $M \gtrsim 600 M_{\odot}$ will remain. This critical mass ratio is comparable to that necessary to avoid ejection from three-body dynamical kicks alone (Gültekin et al. 2004; O'Learv et al. 2006; Gültekin et al. 2006). Since runaway stellar mergers preferentially include the cluster's heaviest stars, which are the precursors to the heaviest black holes in the cluster, the largest black holes used in our calculations may not be present in the cluster.

\section{CONCLUSION}

Our studies indicate that it is a challenge to retain IMBHs during the barrage of $\mathrm{BH}$ mergers expected in an early globular cluster. We find no scenario that guarantees $100 \%$ retention for IMBHs up to $3000 M_{\odot}$ when the interacting $\mathrm{BHs}$ are selected from a reasonable distribution of spins, orientations, and mass ratios. However, the more massive the initial IMBH seed, the better the retention probability. This may indicate that any IMBH observed in globular clusters today would most likely have originated from an early stellar runaway channel.

The results obtained here might be used to test some IMBH formation mechanisms. For example, Fregeau et al. (2006) and Gürkan et al. (2006) find that with a binary fraction above $10 \%$ a single cluster can host multiple runaways, leading to multiple IMBHs. The heaviest two such IMBHs will find their way to the center of the cluster and merge very quickly ( $\sim 1 \mathrm{Myr})$. Because such IMBHs are nearly equal in mass and likely have high spins, they will escape the cluster upon merger. This leaves the cluster without a seed unless a third runaway of sufficient mass occurs. The problem is that the third runaway is often much less massive and therefore difficult to retain, as we have shown.

In fact, since lower mass IMBHs so easily escape globular clusters, if globular cluster observations find that they are not rare, it may be possible to constrain the IMBH merger history, as well. For example, if many low mass IMBHs exist within clusters, we may rule out low mass ratio mergers - this would imply that there is no high mass tail in the BH IMF. Alternatively, the lower the spin, the better the retention as figure 5 shows; if low mass IMBHs are found in large numbers within clusters, and if BHs are found with $>20 M_{\odot}$, we may have to explore ways in which the black holes spin down and align within a gas-poor globular.

Making some very simple assumptions for the primordial globular cluster environment, such as the BH IMF and central density structure, we have estimated that less than 5 globular clusters retain their IMBHs within the Milky Way - even if every one hosted an initial IMBH 
seed. Naturally, there are many uncertainties folded into this estimate, such as the shape of the primordial globular cluster IMF, the degree of mass loss in low metallicity systems and its effect on the BH IMF, and the detailed role that few-body/BH interactions play in shaping early globular cluster structure. As more work is done on these areas, we can revise our estimates using more generic results. For example, if the BH IMF were instead narrowly peaked around $10 M_{\odot}$ (Frver \& Kalogera 2001) and if the seed black hole were a massive supernova remnant, our calculations indicate that about $25 \%$ of the Milky Way globular clusters could retain black holes as small as $\sim 400 M_{\odot}$. An IMBH of a few hundred $M_{\odot}$ may not leave an electromagnetically observable impact on the surrounding globular cluster, as the dynamical effects on the surrounding stars may also be produced by a high binary fraction (Trenti et al. 2007b). However, when stellar mass compact objects merge with these smaller mass IMBHs, they will produce a strong gravitational wave signals that should be detectable with Advanced LIGO (Mandel et al. 2007).

With so many small black holes having been ejected from their host globular clusters, we speculate that 100 rogue black holes are swarming about in the Milky Way halo with masses from $\sim 100-1000 M_{\odot}$, and with velocities mostly on the order of a few hundred $\mathrm{km} \mathrm{s}^{-1}$. The number of rogues could be in the thousands if, as has been suggested, the current globular cluster population is a small fraction of the total number originally created (Aguilar et al. 1988).

Although we have focused on IMBHs in the Galactic globular cluster population, the same processes may occur in other galaxies. Extragalactic ULXs, which may be powered by $\sim 10^{2} M_{\odot}$ IMBHs, are frequently found near, but not in young stellar clusters (e.g., Fabbiano et al. 2001; Liu \& Bregman 2005). Note, though, that the stellar clusters associated with ULXs are not always the dense stellar systems required by the IMBH formation models considered here (Liu et al. 2007). If such IMBHs did form within the nearby clusters, they may be ejected from gravitational wave kicks coming from mergers with stellar-mass black holes, especially as they would merge with the most massive black holes first. While this would explain their separation from the cluster center, it would not explain the fact that ULXs are accreting sources; it is not clear how an IMBH would pick up a companion on its way out of the cluster and is unlikely to retain a stellar companion close enough to overfill its Roche lobe. An IMBH with a stellar companion, however, may be ejected from the host cluster through few-body Newtonian dynamical kicks that harden the binary until it begins accreting (Gültekin et al.|2004; O'Leary et al.|2006; Gültekin et al. 2006; Blecha et al. 2006).

Even if the ejected IMBH is not accreting gas as a ULX, electromagnetic observations may still detect rogue black holes. For instance, if the IMBH were to carry a few massive stars along as it is ejected, our results indicate a kinematically fast subpopulation of massive stars near globular clusters. The ejected black holes may leave a temporary imprint on the globular cluster as well. Since they are ejected from the system impulsively, it is likely that the globular cluster core would temporarily expand. Direct simulations remain to be done to determine how the globular cluster responds to the ejection of an IMBH.

The consequences of these large recoil velocities may also affect SMBH assembly. The most likely candidates for SMBH seeds are $\sim 10^{3} M_{\odot}$ Pop III stellar remnants at redshifts $z \gtrsim 12-20$ (Heger et al. 2003; Volonteri et al. 2003; Islam et al. 2003; Wise \& Abel 2005; Micic et al. 2007). These relic seeds are predicted to form at the centers of low mass dark matter halos $\left(\sim 4 \times 10^{6} M_{\odot}\right)$. As dark matter halos hierarchically merge to assemble the galaxy, the seed black holes sink to the center through dynamical friction and eventually merge. With kick velocities in the range of $\sim 10^{2}-10^{3} \mathrm{~km} \mathrm{~s}^{-1}$, it may also be difficult to retain seed SMBHs in high redshift low mass dark matter halos. We plan to explore black hole retention and possible kick suppression mechanisms at the low mass end of the halo mass function using high resolution cosmological N-body simulations in our next paper.

KHB and KG wish to thank M. Coleman Miller for several very helpful discussions. This work was supported by NSF grant PHY-0354821 to Deirdre Shoemaker. NY is supported by NSF grant PHY-0555628 to Ben Owen.

\section{REFERENCES}

Abel, T., Bryan, G. L., \& Norman, M. L. 2002, Science, 295, 93

Aguilar, L., Hut, P., \& Ostriker, J. P. 1988, ApJ, 335, 720

Baker, J. G., Centrella, J., Choi, D.-I., Koppitz, M., \& van Meter, J. 2006, Phys. Rev., D73, 104002

Baker, J. G., Centrella, J., Choi, D.-I., Koppitz, M., van Meter, J. R., \& Miller, M. C. 2006, ApJ, L93

Baumgardt, H., Hut, P., Makino, J., McMillan, S., \& Portegies Zwart, S. 2003, ApJ, 582, L21

Baumgardt, H., Makino, J., \& Hut, P. 2005, ApJ, 620, 238

Baumgarte, T. W., \& Shapiro, S. L. 1999, Astrophys. J., 526, 941

Belczynski, K., Sadowski, A., Rasio, F. A., \& Bulik, T. 2006, ApJ, 650, 303

Blanchet, L., Qusailah, M. S. S., \& Will, C. M. 2005, ApJ, 635, 508

Blandford, R. D., \& Znajek, R. L. 1977, MNRAS, 179, 433

Blecha, L., Ivanova, N., Kalogera, V., Belczynski, K., Fregeau, J., \& Rasio, F. 2006, ApJ, 642, 427

Bogdanovic, T., Reynolds, C. S., \& Miller, M. C. 2007, ArXiv Astrophysics e-prints

Brügmann, B., Tichy, W., \& Jansen, N. 2004, gr-qc/0312112, to appear in Phys. Rev. Lett.
Campanelli, M., Lousto, C. O., Marronetti, P., \& Zlochower, Y. 2005, Phys. Rev. Lett., 96, 111101

Campanelli, M., Lousto, C. O., Zlochower, Y., \& Merritt, D. 2007a, Ap. J., 659, L5

—. 2007b, Phys. rev. Lett., 98, 231102

—. 2007c, gr-qc/0702133

Damour, T., \& Gopakumar, A. 2006, Phys. Rev. D, 73, 124006

Fabbiano, G. 1989, ARA\&A, 27, 87

Fabbiano, G., \& White, N. E. 2006, Compact stellar X-ray sources in normal galaxies (Compact stellar X-ray sources), 475-506

Fabbiano, G., Zezas, A., \& Murray, S. S. 2001, ApJ, 554, 1035

Favata, M., Hughes, S. A., \& Holz, D. E. 2004, Astrophys. J., 607, L5

Filippenko, A. V., \& Ho, L. C. 2003, ApJ, 588, L13

Fitchett, M. J. 1983, MNRAS, 203, 1049

Fregeau, J. M., Joshi, K. J., Portegies Zwart, S. F., \& Rasio, F. A. 2002, ApJ, 570, 171

Fregeau, J. M., Larson, S. L., Miller, M. C., O'Shaughnessy, R., \& Rasio, F. A. 2006, ApJ, 646, L135

Freitag, M., Rasio, F. A., \& Baumgardt, H. 2006, MNRAS, 368, 121 
Fryer, C. L., \& Kalogera, V. 2001, ApJ, 554, 548

Fryer, C. L., Woosley, S. E., \& Heger, A. 2001, ApJ, 550, 372

Gammie, C. F., Shapiro, S. L., \& McKinney, J. C. 2004, Astrophys. J., 602, 312

Gebhardt, K., Rich, R. M., \& Ho, L. C. 2005, ApJ, 634, 1093

Gerssen, J., van der Marel, R. P., Gebhardt, K., Guhathakurta, P., Peterson, R. C., \& Pryor, C. 2002, AJ, 124, 3270

Gonzalez, J. A., Hannam, M. D., Sperhake, U., Brugmann, B., \& Husa, S. 2007a, gr-qc/0702052

- $2007 \mathrm{~b}$, gr-qc/0702052

Gonzalez, J. A., Sperhake, U., Bruegmann, B., Hannam, M., \& Husa, S. 2006, preprint (gr-qc/0610154)

Gültekin, K., Miller, M. C., \& Hamilton, D. P. 2004, ApJ, 616, 221

-. 2006, ApJ, 640, 156

Gürkan, M. A., Fregeau, J. M., \& Rasio, F. A. 2006, ApJ, 640, L39

Harris, W. E. 1996, AJ, 112, 1487

Heger, A., Fryer, C. L., Woosley, S. E., Langer, N., \& Hartmann, D. H. 2003, ApJ, 591, 288

Heggie, D., \& Hut, P. 2003, The Gravitational Million-Body Problem: A Multidisciplinary Approach to Star Cluster Dynamics (The Gravitational Million-Body Problem: A Multidisciplinary Approach to Star Cluster Dynamics, by Douglas Heggie and Piet Hut. Cambridge University Press, 2003, 372 pp.)

Heggie, D. C., Hut, P., Mineshige, S., Makino, J., \& Baumgardt, H. 2006a, ArXiv Astrophysics e-prints

Heggie, D. C., Trenti, M., \& Hut, P. 2006b, MNRAS, 368, 677

Herrmann, F., Hinder, I., Shoemaker, D., \& Laguna, P. 2007, Class. Quant. Grav., 24, S33

Herrmann, F., Hinder, I., Shoemaker, D., Laguna, P., \& Matzner R. A. 2007, gr-qc/0701143

Islam, R., Taylor, J., \& Silk, J. 2003, Mon.Not.Roy.Astron.Soc., 340, 647

Kennefick, D. 1998, Phys. Rev. D, 58, 064012

Kidder, L. E. 1995, Phys. Rev. D, 52, 821

Koppitz, M., Pollney, D., Reisswig, C., Rezzolla, L., Thornburg, J., Diener, P., \& Schnetter, E. 2007, ArXiv General Relativity and Quantum Cosmology e-prints

Kormendy, J., \& Richstone, D. 1995, ARA\&A, 33, 581

Kroupa, P. 2001, MNRAS, 322, 231

Kulkarni, S. R., Hut, P., \& McMillan, S. 1993, Nature, 364, 421

Liu, J.-F., Bregman, J., Miller, J., \& Kaaret, P. 2007, ApJ, 661, 165

Liu, J.-F., \& Bregman, J. N. 2005, ApJS, 157, 59

Madau, P., \& Rees, M. J. 2001, ApJ, 551, L27
Mandel, I., Brown, D. A., Gair, J. R., \& Miller, M. C. 2007, ArXiv e-prints, 705

Merritt, D., Milosavljevic, M., Favata, M., Hughes, S. A., \& Holz, D. E. 2004, Astrophys. J., 607, L9

Micic, M., Holley-Bockelmann, K., Sigurdsson, S., \& Abel, T. 2007, ArXiv Astrophysics e-prints

Miller, M. C., \& Hamilton, D. P. 2002, MNRAS, 330, 232

O'Leary, R. M., Rasio, F. A., Fregeau, J. M., Ivanova, N., \& O'Shaughnessy, R. 2006, ApJ, 637, 937

Peters, P. C., \& Mathews, J. 1963, Phys. Rev., 131, 435

Portegies Zwart, S. F., Baumgardt, H., Hut, P., Makino, J., \& McMillan, S. L. W. 2004, Nature, 428, 724

Portegies Zwart, S. F., \& McMillan, S. L. W. 2000, ApJ, 528, L17 一. 2002, ApJ, 576, 899

Pretorius, F. 2005, Phys. Rev. Lett., 95, 121101

Ptak, A., \& Colbert, E. 2004, ApJ, 606, 291

Redmount, I. H., \& Rees, M. J. 1989, Comments on Astrophysics, 14,165

Rees, M. J., \& Volonteri, M. 2007, ArXiv Astrophysics e-prints

Roberts, T. P., \& Warwick, R. S. 2000, MNRAS, 315, 98

Schneider, R., Ferrara, A., Natarajan, P., \& Omukai, K. 2002, ApJ, 571, 30

Shibata, M., \& Shapiro, S. L. 2002, Astrophys. J. Lett., 572, L39

Sigurdsson, S., \& Hernquist, L. 1993, Nature, 364, 423

Sopuerta, C. F., Yunes, N., \& Laguna, P. 2006, Phys. Rev. D, 74, 124010

—. 2007, ApJ, 656, L9

Spitzer, L. 1987, Dynamical evolution of globular clusters (Princeton, NJ, Princeton University Press, 1987, 191 p.)

Thorne, K. S. 1980, Rev. Mod. Phys., 52, 299

Tichy, W., \& Marronetti, P. 2007, gr-qc/0703075

Trenti, M. 2006, ArXiv Astrophysics e-prints

Trenti, M., Ardi, E., Mineshige, S., \& Hut, P. 2007a, MNRAS, 374,857

-. 2007b, MNRAS, 374, 857

Trenti, M., Heggie, D. C., \& Hut, P. 2007c, MNRAS, 374, 344

Ulvestad, J. S., Greene, J. E., \& Ho, L. C. 2007, ApJ, 661, L151

van der Marel, R. P. 2004, in Coevolution of Black Holes and Galaxies, ed. L. C. Ho, 37-+

Volonteri, M., Haardt, F., \& Madau, P. 2003, ApJ, 582, 559

Webbink, R. F. 1985, in IAU Symposium, Vol. 113, Dynamics of Star Clusters, ed. J. Goodman \& P. Hut, 541-577

Whitbeck, D. 2006, PhD thesis, Department of Physics, University Park

Wise, J. H., \& Abel, T. 2005, ApJ, 629, 615 